\title{
Reducing Suboptimal Bowel Preparation
}

\section{Colleen A Sawyer RN, Pallavi Kour RN and Abigail Mitchell RN*}

D’Youville College, Buffalo NY, USA

*Corresponding author: Abigail Mitchell RN, D’Youville College, Buffalo NY, USA, Email: mitchela@dyc.edu

\section{Mini Review}

Volume 4 Issue 4

Received Date: July 02, 2021

Published Date: July 14, 2021

DOI: $10.23880 /$ jqhe-16000232

\section{Abstract}

Achieving adequate bowel cleansing is essential to ensuring safe and effective colonoscopy. Yet, among hospitalized patients, suboptimal bowel preparation is a common problem leading to diagnostic delay, increased cost, and patient dissatisfaction. All patients, inpatient and outpatient, presenting with inadequate bowel preparation prior to procedure require repeat colonoscopy sooner than needed with successful bowel preparation.

Keywords: Bowel Preparation; Quality Improvement; Inpatient Colonoscopy; Education Intervention; Nursing Care Impact

\section{Introduction}

In 2015, an estimated 11 million colonoscopies were completed in the United States. By 2017, this number increased to 19 million and the number of colonoscopies completed annually is projected to continue this ascent because of the aging population [1]. Colonoscopy is the preferred procedure for colorectal cancer screening because it allows direct visualization and of colonic mucosa and provides the opportunity to remove potentially precancerous polyps [2]. Additionally, colonoscopy procedures are widely performed to evaluate and treat symptoms such as chronic diarrhea, unexplained abdominal pain, weight loss, anemia, and suspected acute lower gastrointestinal bleeding [1]. Importantly, a high quality, efficacious colonoscopy depends upon adequate bowel cleansing to facilitate the visualization of the mucosal surface of the colon.

Even though successful colonoscopies require adequate preparation, between $18 \%$ and $30 \%$ of all patients undergoing colonoscopy procedures may not achieve needed optimal bowel cleansing [3]. Worse yet are rates of inadequate bowel preparation found among hospitalized patients. Despite receiving the support and supervision of nurses, suboptimal bowel preparation among hospital inpatients occurs from $20 \%$ to $50 \%$ of the time [4]. All patients, inpatient and outpatient, presenting with inadequate bowel preparation prior to procedure require repeat colonoscopy sooner than needed with successful bowel preparation. Suboptimal bowel cleansing increases the duration of colonoscopy procedures and risk for complications, such as bowel perforation [3]. Inadequate cleansing decreases lesion detection, patient satisfaction, and the rate of completed procedures [5]. Among hospitalized patients repeat colonoscopies, often caused by lack of comprehension of instructions and noncompliance, contribute to increased length of stay. For all these reasons, improving the quality of inpatient bowel preparations positively influences health care costs, quality of care, and patient safety and satisfaction.

\section{Cost}

Gastrointestinal (GI) diseases account for substantial morbidity, mortality among United States citizens and contribute substantially to health care costs. Annual total expenditures for GI diseases is estimated to be $\$ 135.9$ billion dollars [1]. In 2014, GI hemorrhage alone, accounted for more than 500,000 hospitalizations and approximately 2.2 million days of hospitalization. As previously stated, investigation and treatment of GI hemorrhage is a leading indication for colonoscopy among hospitalized patients. Additionally, GI hemorrhage is a significant cause of hospital readmission 
[1]. The increased economic burden associated with poor bowel preparation is also significant and causes both direct and indirect costs assumed by patients and providers. Poor bowel preparation among hospitalized patients may increase the costs by $12 \%$ to $22 \%$ [6]. Further, a single-center study suggests acute care hospital savings of $\$ 150.000$ to $\$ 200,000$ dollars annually by reducing the number of repeat procedures due to poor inpatient colon cleansing [7].

\section{Importance of Adequate Bowel Cleansing}

A colonoscopy is a procedure performed by a physician to examine the lower gastrointestinal (GI) tract. Colonoscopies are completed using an endoscope, which consists of a long, narrow flexible tube equipped with its own light source and camera. In addition to allowing visualization and imaging of the colon from the cecum to the rectum, the flexible scope contains an auxiliary water channel, an air and water nozzle, and an instrument channel for biopsy forceps or other instruments to remove polyps, take biopsies, cauterize bleeding or perform other procedural therapies [8]. For this reason, providers use colonoscopy to perform both diagnostic and therapeutic interventions.

The US Multi-Society Task Force of Colorectal Cancer (MSTF), which represents the American College of Gastroenterology, the American Gastroenterological Association, and the American Society for Gastrointestinal Endoscopy, recommends colonoscopy as the preferred, first tier screening procedure for colorectal cancer, for surveillance of patients with previously detected precancerous polyps or lesions, and to detect dysplasia in persons with inflammatory bowel diseases of the colon [2]. In fact, it is estimated that routine screening colonoscopies may prevent up to $65 \%$ of all colorectal cancers [9]. In addition to investigations related to inflammatory bowel disease and colorectal cancer, colonoscopy is used to diagnose and treat unexplained abdominal pain, anemia, and lower gastrointestinal bleeding [10].

The success of colonoscopy is fully dependent on the quality of bowel preparation prior to procedure. Not surprisingly, poor bowel cleansing is the leading cause of failed colonoscopies [7]. Yet, multiple studies indicate that between $18 \%$ to $30.5 \%$ of patients undergoing colonoscopy have suboptimal bowel cleansing [11]. Compared to outpatient populations, hospitalized patients typically have poorer quality bowel preparations. As a matter of fact, up to $60 \%$ of inpatient colonoscopies are associated with inadequate bowel preparation [12]. Specifically, one major retrospective study, reviewing the electronic reports of nearly 30,000 colonoscopies occurring over a 10-year period, found the inpatient setting among the most prominent factors associated with suboptimal bowel preparation [13].

\section{Factors Impacting Quality of Bowel Preparation}

Colonoscopy requires complete cleansing of the colon. A variety of bowel cleansing regimes are commonly in use, but most have in common a clear liquid diet for 24 to 48 hours before the procedure and some type of laxative preparation. A thorough understanding of stool consistency, color, and presence of sediment is helpful to assess the level of bowel cleansing preparation. Quality of bowel cleansing depends on the ability to visualize the mucosa of the colon once retained stool and fluid have been eliminated. Best practice recommends endoscopists include the quality of preparation for each colonoscopy; however there is no standardized definition for terms used to characterize bowel preparations [6].

\section{Nonmodifiable Risk Factors}

Many research studies have identified barriers that impact patient's ability to successfully complete bowel preparations for colonoscopy. These studies consistently find an association with older age and diabetes and inadequate bowel preparation. Increased age is associated with multiple comorbidities, such as chronic constipation, and multiple surgeries while diabetes decreases gut motility increasing the transit time for bowel preparation [12]. Additionally, Ydlapati, et al. identified African American race, and tricyclic antidepressant use as a risk factors for inadequate inpatient preparation.

\section{Modifiable Risk Factor}

One modifiable risk factor for inadequate preparation is the selection of bowel preparation solution used for individual patients. Several different bowel preparations are in current use. The ideal agent should be convenient to use, cleanse the bowel, and be well tolerated with minimal side effects. The common bowel solutions administered before colonoscopy are polyethylene glycol-electrolyte lavage solutions (PEGELS), oral sulfate solutions, sodium picosulfate, or sodium phosphates with magnesium citrate [14]. Since they are isoosmotic, PEG-ELS solutions such as GoLytely, are considered the preferred agents to use in patients less likely to tolerate fluid shifts, such as patients with renal insufficiency, congestive heart failure, and advanced liver disease. Thus, PEG-ELS solutions are currently the most frequently administered agent used for preparations for hospitalized patients [14]. Further, a recent prospective and randomized pilot study compares the efficacy of high-volume PEG-ELS solutions to medium and low volume preparation regimens among hospitalized patients undergoing colonoscopy procedures, found no statistical difference in the adequacy of bowel cleansing. A later 2018 study, also conducted 
by Yadlapati, demonstrated a significance increase in the adequacy of bowel preparation among hospitalized patients using a split-bowel preparation. However, this study initiated the use of an order set for split bowel preparation, automated orders for rescue medications, and nursing bowel preparation checks. Since this was an interdisciplinary effort to reduce repeated colonoscopy procedures the authors recognize that there is no way to recognize the independent impact of the split dose regimen [15].

Pre-procedure nausea and vomiting which prevents consumption of the preparation agent and afternoon colonoscopies other modifiable determinants associated with inadequate bowel preparation [16]. Colonoscopy procedures occurring before noon are associated with enhanced quality compared to afternoon procedures [12]. Runway time is defined as the interval between the last ingestion of the preparation and the start of the colonoscopy procedure. Interestingly, bowel preparation quality is inversely related to this interval [12]. This phenomenon helps explain the recommendation for split dose preparations noted in clinical practice guidelines [14]. Thus, in hospital settings important considerations include identifying and treating underlying nausea to facilitate compliance with preparation and completing colonoscopy procedures within optimal runway time.

\section{Nursing}

Interestingly, education interventions are notrestricted to patients. The importance of providing sufficient information to patients and nurses about colonoscopy and following the recommended schedule of bowel preparation cannot be overemphasized [12]. One recent analysis of possible reasons for inadequate bowel preparations in a university hospital identified communication failures among health care teams, lack of step-by-step-instructions for the nursing staff, and nurses' lack of knowledge as contributing factors to suboptimal bowel cleansing [17]. Consumption of the large volume of preparation solution is a difficult for patients. For this reason, it is important nurses understand the cleansing process and its importance, so they can encourage patient persistence and vigilance with preparation administration.

\section{Educational Interventions to Improve Bowel Preparation}

Current practice guidelines recommend healthcare professionals provide oral and written patient education instructions for all components of the colonoscopy preparation and emphasize the importance of compliance [14]. While many intrinsic factors cannot be changed with interventions, patient compliance can be improved through enhanced communication. Nearly $20 \%$ of patients with poor bowl preparation failed to follow preparation instructions [11]. Patient education is crucial because the inability to follow instructions on consuming bowel preparation is an independent predictor of inadequate bowel preparation [12]. Further, education is a viable and low-cost strategy for quality improvement for colonoscopy preparation [18].

Patient centered bowel preparation has been explored in numerous studies to improve patient outcomes and has yielded mixed results [19]. In the current research, enhanced educational interventions, such as videos and educational booklets with visual aids, are more concise and intuitive than traditional instructions. Plus, educational videos are suitable for patients with a low reading ability and across larger ranges of educational and health literacy levels [3]. Further, the use of novel educational materials on pre-colonoscopy preparation consistently results in better bowel preparation scores than those achieved using conventional instruction [14]. Finally, in their systematic review of strategies to improve inpatients' quality of bowel preparation for colonoscopy Gkolfakis, Tziatzios, Papanilolau, and Triantafillou (2019) conclude educational interventions are more significant than bowel preparation solution selection in improving inpatient's bowel preparation quality. Providing inpatients with detailed instructions is an example of an evidence-based practice that is often overlooked among hospitalized patients. Knowledge about adequate preparations, hardships with completing the cleansing regime, and multiple patient related factors impact the efficacy of preparation. Park, et al. [20] designed a bowel preparation video emphasizing the importance of adhering to bowel preparation instructions. , educational interventions have been shown to improve patient satisfaction [17]. Thus, implementing use of enhanced educational materials likely improves the quality of inpatient bowel preparation and increases patient satisfaction [21-23].

\section{Conclusion}

Adequate bowel preparation is essential for optimal procedure outcomes. Findings from the review of literature reveal optimal assessment of the large bowel with colonoscopy requires minimal fecal contamination. Yet, most hospitalized patients continue to have suboptimal bowel preparation for colonoscopy procedures. An inadequately prepared bowel leads to missed diagnosis, need for repeat procedures and investigations causing a significant financial impact to both the patient and healthcare systems [7]. Education is a common theme of successful quality improvement programs in gastroenterology [18]. Additionally, providing inpatients with detailed instructions is an example of an evidencebased practice that is often overlooked among hospitalized patients. Knowledge about adequate preparations, hardships with completing the cleansing regime, and multiple patient related factors impact the efficacy of 
preparation. Educational interventions have been shown to improve patient satisfaction [17]. Thus, implementing use of enhanced educational materials likely improves the quality of inpatient and outpatient bowel preparation and increases patient satisfaction $[24,25]$.

\section{References}

1. Peery AF, Crockett SD, Murphy CC, Lund JL, Dellon ES, et al. (2019) Burden and cost of gastrointestinal, liver, and pancreatic diseases in the United States: Update 2018. Gastroenterology 156(1): 254-272.

2. Rex DK, Bolanad R, Dominitz JA, Giardiello F, Johnson DA, et al. (2017). Colorectal cancer screening: Recommendations for physicians and patients from the U.S. Multi-Society Task Force on colorectal canacer. Gastrointestinal Endoscopy 86(1): 18-33.

3. Liu Z, Zhang MM, Li YY, Li X, Li YQ (2017) Enhanced education for bowel preparation before colonoscopy: A state of the art review. Journal of Digestive Diseases 18(2): 84-91.

4. Corliss JA (2017) Effect of low-volume split dose purgative on the quality of bowel prep for colonoscopy on the hospitalized patient. Gastroenterology Nursing 40(6): 448-457.

5. Catalan CC, Laura E, Almudena N, McAngels M, Avonello M (2019) Bowel prepararation for inpatient colonoscopy: A quality evaluation. Gastrointestinal Nursing 17(4): 4450 .

6. Rex DK, Schoenfeld PS, Cohen J, Pike IM, Adler DG, et al. (2015). Quality Indicators for Colonoscopy. The American Journal of Gastroenterology 110: 72-90.

7. Gardezi SA, Tibbatts C (2017) Improving bowel preparation for colonoscop in a cost effective manner. BMJ Quality 6(1): 1-5.

8. American College of Gastroenterology (2019) Colonoscopy.

9. Miller SJ, Iztkowitz SH, Redd WH, Thompson HS, Valdimarsdottir HB, et al. (2015) Colonoscopy-specific fears in African Americans and Hispanics. Behavioral Medicine 41(2): 41-48.

10. Argyopoulos SK, Mahmood SK, Campbell EJ, Richter JM (2018) Improving the quality of inpatient bowel preparation for colonoscopies. Digestive Diseases and Sciences 63: 338-344.

11. Guo X, Yang Z, Zhao L, Leung F, Luo H, et al. (2017)
Enhanced instructions improve the quality of bowel preparation for colonoscopy: A meta-analysis of randomized control trials. Gastrointestinal Endoscopy 85(1): 90-97.

12. Ahmed Z, Zafar Y, Sarvepalli S, Burke C (2019) Determinants of bowel preparation quality for colonoscopy: The role of modifiable and nonmodifiable factors. Gastroenterology and Endoscopy News pp: 1-8.

13. Baker FA, Mari A, Nafrin S, Suki M, Ovadia B, Gal O, Kopelamn Y (2019) Predictorsand colonoscopyoutcomes of inadequate bowel cleansing: A 10-year experience in 28,725 patients. Annals of Gastroenterology 32: 457462.

14. Johnson DA, Barkun AN, Cohen LB, Dominitz JA, Kaltenbach T, et al. (2014) Optimizing adequacy of bowel cleansing for colonoscopy: Recommendations from the US Multi-Society Task Force on colorectal cancer. The American Journal of Gastroenterology 109(10): 15281545.

15. Gluskin AB, Keswani RN, Johnston ER, Gregory DL, Cyrus R, et al. (2016) The Nursing Bowel Preparation Asseesment Tool (NBPAT) is a reliable and accurate inpatient colonoscopy clnical desicison support tool. Gastrointestinal Endoscopy 83(5S): Sa1038.

16. Yadlapati R, Johnston ER, Gregory DL, Ciolino JD, Cooper A, et al. (2015) Predictors of inadequate inpatient colonoscopy preparation and its association with hospital length of stay and costs. Digestive Diseases and Sciences 60(11): 3482-3490.

17. Chambers K, Whiteman K, Stephens K, Goodloe L, Kirsteen H (2016) Improving inpatient colonoscopy preparation in a university hospital. Gastroenterology Nursing 39(2): 86-94.

18. Reynolds C, Esrailian E, Hommes D (2018) Quality improvement in gastroenterolgy: A systematic review of practical interventions for clinicians. Digestive Diseases and Sciences 63: 2507-2518.

19. Gkolfakis P, Tziatzios G, Papanikolaou IS, Triantafyllou K (2019) Strategies to improve inpatient' quality of bowel preparation for colonoscopy: A systematic review and meta-analysis. Gastroenterology Research and Practice 2019: 1-15.

20. Park JS, Kim MS, Kim H, Kim SI, Shin CH, et al. (2016) A randomized controlled trial of an educational video to improve quality of bowel preparation for colonoscopy. BMC Gastrtoenterology 16(64): 1-8. 
21. Calderwood AH, Jacobsen BC (2010) Comprehensive validation of the Boston Bowel Preparation Scale. Gastrointestinal Endoscopy 72(4): 686-692.

22. Ergen WF, Pasricha T, Hubbard FJ, Higginbotham T, Givens T, et al. (2016) Providing hospitilized patients with an educational booklet increases the quality of colonsocopy bowel preparation. Clinical Gastroenterology and Hepatology 14(6): 858-864.

23. Lee YJ, Kim ES, Park KS, Cho KB, Jang BK, et al. (2015) Education for ward nurses innfluences the quality of inpatient's bowel preparation for colonoscopy. Medicine 94(34): 1-9.

24. Miller K, Briody C, Casey D, Kane JK, Mitchell D, et al. (2016) Using the comprehensive unit-based safety program model for sustained reduction in hospital infections. American Journal of Infection Control 44: 969-976.

25. Shah Khan SM, Cumberledge J, Reynolds GJ (2017) Using the plan-do-study-act approch to improve inpatient colonoscopy preparation. BMJ Open Quality pp: 1-6. 